\title{
Assessment of fog and rain induced-attenuation on terrestrial FSO links
}

\author{
M. O. Ajewole ${ }^{1}$, P. A. Owolawi ${ }^{2}$, J. S. Ojo ${ }^{3 *}$, R. M. Adetunji ${ }^{4}$ \\ ${ }^{1}$ Department of Physisc, Federal University of Technology, Akure, P. M. B. 704, Akure, Nigeria \\ ${ }^{2}$ Department of Computer Systems Engineering, Building: 19, Office No 204, Soshanguve South Campus \\ Tshwane University of Technology, Pretoria, South Africa \\ *Corresponding author, e-mail: ojojs_74@futa.edu.ng
}

\begin{abstract}
Launching into the next generation of wireless communication network (5G network) requires secure high data rate, high speed and huge bandwidth links. With the tremendous increase in broadband users, the existing communication systems such as radio frequency $(R F)$ and microwave links cannot meet up with the challenges due to their link interference and low bandwidth. A current technology that promises such requirements and more is Free Space Optical (FSO) communication. The FSO basically involves the transmission of signal-modulated optical radiation from a transmitter to a receiver through the atmosphere or outer space. It is designed to complement the traditional fibre optical communication links. However, location-variant atmospheric channel degrades the quality and performance of an FSO system under severe atmospheric conditions. This paper attempts to assess both fog-and rain-induced attenuation on the performance of FSO link in a terrestrial terrain using measured visibility and rain rate data at Akure, Nigeria. 5-year (2012-2016) archived visibility data and measured rain rate data of 1-minute integration time obtained from Nigerian Meteorological Agency (NIMET) and the Department of Physics, Federal University of Technology, Akure respectively, were used to compute the fog-and rain-induced specific attenuations using Kruse and Carboneur models. The performance of the FSO system was analyzed through link margin by using the parameters of a commercial optical transceiver, Terescope 5000. Findings from this work will be useful for FSO system design in the area.
\end{abstract}

Keywords: atmospheric loss, Free Space Optics (FSO), link margin, hydrometeors, probability

Copyright $@ 2019$ APTIKOM - All rights reserved.

\section{Introduction}

The advancement in communication technology, internet, calls, data, video teleconferencing, radio and video streaming among others now travel hundreds of miles in a short period when converted and sent as light through plastic or glass strands between two terminals. This can be effectively achieved through a technology known as fibre optics communication. Radio frequency (RF) and microwave links have also been known to provide users mobility within a signal coverage region. However, these two systems have limitations for current secure high data rate and bandwidth demands of modern broadband communication applications. RF links have problems such as congested spectrum, low transmission data rate, expensive license acquisition, data insecurity, high cost of installation and accessibility [1]. Optical fibre also have limitations such as high installation cost, high cost of equipment, long period of installation, long period of right-of-way acquisition to mention but few.

The short-comings associated with fibre optics and RF has led to the emergence, investigation and deployment of optical wireless communication (OWC) as an alternative communication technology. In outdoor use, OWC is referred to as FSO communication system. FSO communication is a line-of-sight (LOS) communication technology whereby the signals transmitted (data, voice or video) are in form of optical radiation (laser) through unguided atmospheric channels between two transceivers [1]. FSO has numerous advantages that include being cost effective, license-free, and having high bandwidth, high data rate and easy installation. This technology has been recently adopted for a variety of applications. Among the applications includes last-mile network, teleconferencing, inter-satellite links optical fibre backup, campus internet interlinks [1].

A typical FSO system has three segments namely: the transmitter, the atmospheric channel, and the receiver. Of the three segments, the atmospheric channel is the most unpredictable and locationvariant. Despite the overwhelming qualities of the technology, it suffers degraded performance under 
different abnormal atmospheric conditions. Among the conditions mitigating FSO channels' availability and reliability is the presence of fog, rain, snow, haze, cloud, midst, etc. Among the hydrometeors, fog is the most deleterious to an FSO system [2]. Studies have shown that attenuation in an FSO system could have peak value as high as $480 \mathrm{~dB} / \mathrm{km}$ under dense maritime fog [3]. In this paper, the impairments caused by fog and rain on FSO links system in the study area are presented.

Hydrometeor is the general term referring to the products of condensation of water vapour in the atmosphere. It is usually observed as rain, hail, ice, fog, cloud or snow. Of the hydrometeors, fog poses the greatest threat to the FSO links' availability and reliability. Since the current study focuses on fogand rain-induced attenuations on an FSO link system, detailed account on only these two hydrometeors are presented. Fog is composed of an aggregate of very fine water droplets and ice in the air close to the surface of the earth [2]. Fog formation is dependent on the meteorological state of the atmosphere close to ground. The meteorological conditions involved are relative humidity, temperature and dew point. Fog formation results whenever there is a significant increase in the relative humidity above $85 \%$, and the difference between temperature and dew point is less than or equal to $3{ }^{\circ} \mathrm{C}$ [4]. There are two basic types of fog namely: radiation fog and maritime fog. While radiation fog is formed by temperature inversion, maritime fog is formed due to the movement of damp air over a cold or water surface [1].

An obvious effect of fog event is that it blurs vision by reducing the clarity and range of view. The meteorological term used to describe the extent of view in a foggy atmosphere is visibility. Visibility is a linear measurement of distance (in $\mathrm{km}$ ) of the meteorological visual range (MVR) at which an object reduces to a defined percentage of its original size [1]. Meteorologically speaking, an atmosphere is regarded as being foggy when the visibility is just less than $1 \mathrm{~km} \mathrm{[2].} \mathrm{Visibility} \mathrm{is} \mathrm{measured} \mathrm{with} \mathrm{a}$ transmissometer which is usually installed close to the ground in a wide open lateral terrain. Rain is the condensation of water falling downwards from a cloud. It is a natural phenomenon that has both temporal and spatial variability. Its effects, especially those with high rain rate, produce an attenuating effect which contributes to the signal degradation on FSO links or outright unavailability of radio waves (absorption) with frequencies above $10 \mathrm{GHz}$ on microwave links. There are basically two types of rain namely: convective rain and stratiform rain. In most cases, rain occurs in the form of cells which are complex mixture of convective and stratiform rains in the tropics. Such mixed rain type accounts for about $70 \%$ or more of the total rain in most cases [5].

Convective rains have three characteristic features: high intensity rainfall, short durations (usually less than an hour) and small coverage areas (few kilometers). Two basic sub-divisions of convective rain are shower and thunderstorm. Stratiform rain also has three characteristic features: medium and low intensity rainfall, long duration (several hours) and wider coverage area (several kilometers). Two sub-divisions of this type of rain are drizzle and widespread. The rest of this paper is organized as follows: Section 2 presents methodology adopted. Section 3 presents the results and discussion, while section 4 is the concluding remarks.

\section{Research Method}

The section presents information on the methodology adopted in this work

\subsection{Study Area and Data}

The study site is Akure, Ondo State, Southwestern Nigeria. It has a latitude of $7.17 \mathrm{oN}$ and longitude of $5.18 \mathrm{o} \mathrm{E}$, and is $315 \mathrm{~m}$ above sea level. The climate in Akure is tropical, with more high intensity rainfalls observed during the wet season months than the dry season months. Two sets of data were used in this study. The first data is a 5-year archived visibility data (January, 2012-December, 2016) which was obtained from Nigerian Meteorological Agency (NIMET) for the study location. The second data is a 5-year rain rate (January, 2012-December, 2016) obtained from the weather station in the Department of Physics, Federal University of Technology, Akure, Nigeria.

\subsection{Fog-induced Specific Attenuation Model based on Visibility}

Specific attenuation refers to signal loss per unit length. Thus, fog-induced specific attenuation $(\mathrm{dB} / \mathrm{km})$ by Kruse model is expressed as [1]:

$$
\beta_{\text {spec }}=\frac{10 \log T_{\text {th }}}{V(k m)}\left(\frac{\lambda}{\lambda_{0}}\right)^{-q}
$$


where $V$ is the visibility in $\mathrm{km}, T_{t h}$ is the transmittance threshold, $\lambda(\mathrm{nm})$ is the optical wavelength, and $\lambda_{0}$ is the maximum spectrum wavelength of the solar band $(550 \mathrm{~nm})$. For this model, the values of the index ' $q$ ' in (1) depend on visibility ranges as:

$$
q=\left\{\begin{array}{llr}
1.6 & \text { if } & V>50 \mathrm{~km} \\
1.3 & \text { if } & 6 \mathrm{~km}<V<50 \mathrm{~km} \\
0.585 V^{1 / 3} & \text { if } & 0 \mathrm{~km}<V<6 \mathrm{~km}
\end{array}\right.
$$

Terrestrial FSO communication makes use of $2 \%$ transmittance threshold. As such, the value was used with the archive visibility data for five optical wavelengths of 650, 750, 850, 950 and $1550 \mathrm{~nm}$.

\begin{tabular}{cll}
\hline \multicolumn{1}{c}{ Device Parameters } & \multicolumn{1}{c}{ Value } \\
\hline \multicolumn{1}{c}{ Model } & TS5000/4E1 \\
& Standard P. N. & TS5000/4E1/V \\
& & Ethernet/(10 Mbps $)$ \\
Typical Application/Data Protocol & Fast Ethernet/(1-155 Mbps $)$ \\
& & Gigabit Ethernet Escon/(622 Mbps $)$ \\
& Light Source & Fibre Channel and Others/(100-1500 Mbps $)$ \\
Transmitter & Wavelength & $3 \times$ Lasers \\
& Total Output Power & $830-860 \mathrm{~nm}$ \\
& Beam Divergence Angle & $2 \mathrm{~mW}$ \\
& Transmit Aperture Diameter & $3 \mathrm{~cm}$ \\
& Detector & APD \\
& Field of view & $2 \mathrm{mrad}$ \\
Receiver & Sensitivity & $-46 \mathrm{dBm}$ \\
& Receive Aperture Diameter & $10 \mathrm{~cm}$ \\
\hline
\end{tabular}

\subsection{Probability Distribution of Encountering Fog-induced Specific Attenuation at Different Wavelengths and Transmittance Thresholds Values}

Probability is a statistical concept that studies the chance or propensity of occurrence of an event. More than just a numerical entity that counts the number of occurrence of an event or experimental outcome, probability is a rational entity that show cases the relative chance of occurrence of an event under consideration. Given a sample space $\mathrm{n}(\mathrm{S})$ and number of experimental outcome $\mathrm{n}(\mathrm{E})$, the percentage probability of occurrence of the outcome is given as:

$$
P(E)=\frac{\mathrm{n}(\mathrm{E})}{\mathrm{n}(\mathrm{S})} \times 100 \%
$$

The percentage probability of encountering varying degree of fog-induced specific attenuation at different wavelength and transmittance threshold values was computed using (3). $E$ is the event of foginduced specific attenuation within a defined range, $n(E)$ is the corresponding numerical value, while $n(S)$ is defined as sample space for the total number of attenuation values.

\subsection{Rain-induced Specific Attenuation}

Although fog is the major factor causing signal degradation of optical radiation in an FSO channel, rain also contributed some degree of degradation to the link. In this study, attenuation due to rain was considered due to its predominance in the weather pattern of the study area. The atmospheric loss due to rain, $\propto_{\text {rain }}$ in terms of its precipitation rate $R_{p t}$, is expressed as [9]:

$$
=1.076 \times R_{p t}^{0.67}(\mathrm{~dB} / \mathrm{km})
$$

\subsection{Link Margin Analysis}

For a reliable communication system, one crucial task of a good communication system designer is to carry out link power budget analysis. This analysis simply entails the inclusion of all known powerloss sources into the transmitting power, with the intention of ensuring a certain minimum power reaches the receiver on the other end of the link. In this study, the loss sources considered in the link budgeting were fog- and rain-induced specific attenuations. Link margin is the difference between the receiver's sensitivity and the expected minimum received power. If the link margin is lower than a minimum value 
required by a receiver to function properly (receiver sensitivity), the system will be unavailable. The link margin is defined as [8]:

$$
L M(\mathrm{~dB})=P_{t}+\left|S_{r}\right|-A t t_{G e o}-\Sigma A t m_{\text {Losses }}
$$

where $P_{t}$ is the transmitted power in $\mathrm{d} B m,\left|S_{r}\right|$ is the absolute value of the receiver sensitivity in $\mathrm{dBm}$, $A t t_{\text {Geo }}$ is the geometric loss $\mathrm{dBm}$, and $\Sigma \mathrm{Atm}_{\text {Losses }}$ is the summation of the various atmospheric losses in $\mathrm{dB}$.The geometric loss in decibel is thus given as [1]:

$$
A t t_{G e o}=-20 \log \left[\frac{d_{r}}{\left(d_{t}+\theta_{b} L_{d}\right)}\right]
$$

where $d_{r}$ and $d_{t}$ are the receiver and transmitter aperture diameters respectively (in $\mathrm{cm}$ ), $\theta_{b}$ is the beam divergence angle (in mrad), and $L_{d}$ is the FSO link distance (in $\mathrm{km}$ ). In this study, a Table 1 TereScope ${ }^{\circledR}$ 5000 Technical Specifications [9]. commercial optical transceiver (TereScope 5000) as presented in Table 1 has been adopted to assess the realistic link power budgeting for the study area. The first scenario considers only geometric loss and fog-induced loss such that (5) becomes:

$$
L M_{G e o \& F o g}(\mathrm{~dB})=P_{t}+\left|S_{r}\right|-A t t_{G e o}-A t t_{F o g}
$$

where

$$
A t t_{F o g}=10 \log \left(e^{-\beta_{\text {spec }} \times L_{d}}\right)
$$

By substituting equation (6) and equation (8) into equation (7), it yields:

$$
L M_{\text {Geo\&Fog }}(\mathrm{dB})=P_{t}+\left|S_{r}\right|-20 \log \left[\frac{d_{r}}{\left(d_{t}+\theta_{b} L_{d}\right)}\right]-\left[10 \log \left(e^{-\beta_{\text {spec }} \times L_{d}}\right)\right]
$$

The fog-induced loss was computed at five optical wavelengths of 650, 750, 850, 950 and 1550 $\mathrm{nm}$, link distances $\left(L_{d}\right)$ of $1,2,3,4,5, \ldots, 10 \mathrm{~km}$ and $2 \%$ transmittance threshold at a visibility value of 5 $\mathrm{km}$. The selected visibility value represents a frequent visibility range for the study location. The second loss scenario considers only geometric loss and rain-induced loss such that (5) becomes:

$$
L M_{\text {Geo\&Rain }}(\mathrm{dB})=P_{t}+\left|S_{r}\right|-A t t_{G e o}-A t t_{\text {Rain }}
$$

where

$$
A t t_{\text {Rain }}=\left(1.076 \times R_{p t}^{0.67}\right) \times L_{d}
$$

By substituting (6) and (11) into (10), it yields:

$$
L M_{\text {Geo\&Rain }}(\mathrm{dB})=P_{t}+\left|S_{r}\right|-20 \log \left[\frac{d_{r}}{\left(d_{t}+\theta_{b} L_{d}\right)}\right]-\left[\left(1.076 \times R_{p t}{ }^{0.67}\right) \times L_{d}\right]
$$

where all parameters retain their previous definitions, and $L_{d}$ is the link distance $(\mathrm{km})$. The rain-induced loss was computed at rain rates of 5, 10, 15, 20, 50, 100 and $200 \mathrm{~mm} / \mathrm{hr}$. These values were selected from the rain rate data representing both convective and stratiform rainfall type observed in the study area. The computations were carried out over the same set of link distances.

\section{Results and Discussion}

Figure 1 presents the estimated specific attenuation at 650,850 and $1550 \mathrm{~nm}$ wavelengths based on $2 \%$ transmittance threshold against daily visibility over a year based on Kruse model. Three peak values of specific attenuation were observed on three foggy atmospheric conditions with visibility value as low as $0.3 \mathrm{~km}$. These values occurred at the 35th, 269th and 317th days of the year with maximum specific attenuation values of about 122.14, 109.96 and 86.91 at 650,850 and $1550 \mathrm{~nm}$ wavelengths respectively. The estimated specific attenuation decreases by about $9.97 \%$ from the value at $650 \mathrm{~nm}$ to that at $850 \mathrm{~nm}$. However, from $650 \mathrm{~nm}$ to $1550 \mathrm{~nm}$, attenuation values decrease by up to about $28.84 \%$.

APTIKOM J. CSIT Vol. 4, No. 1, 2019: $37-44$ 
Another foggy event of lower intensity was observed on the 351st day of the year with a visibility value of $0.5 \mathrm{~km}$.

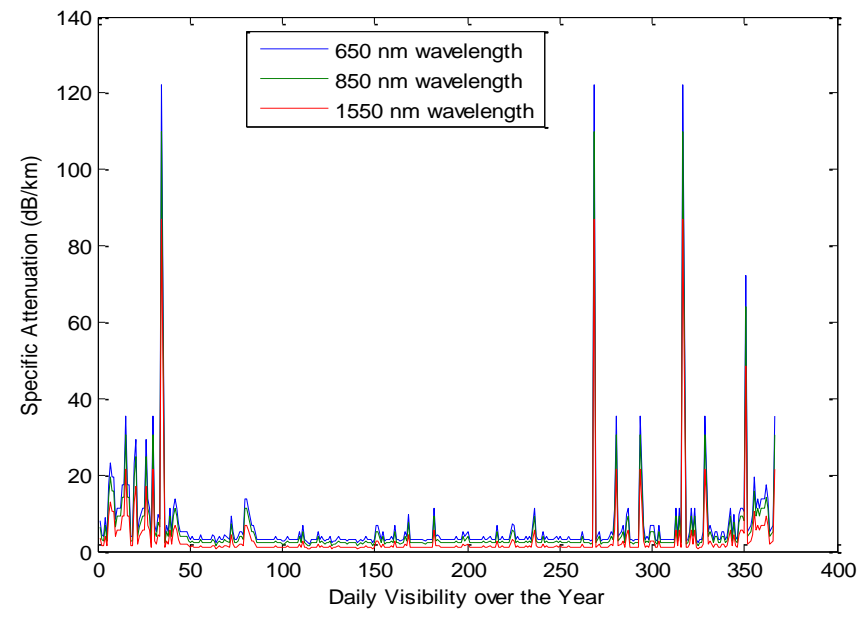

Figure 1. Estimated Specific Attenuation using Kruse Model at 2\% Transmittance Threshold

It is also worthy to assess the probability distribution of grouped specific attenuation occurrence at the study area. This is necessary to deduce the time of the year at which the optical link remains unavailable [4]. The result on estimated specific attenuation was used to compute the percentage probability of occurrence of grouped specific attenuation values (with a class width of 5) at five optical wavelengths of $650,750,850,950$ and $1550 \mathrm{~nm}$, and $2 \%$ transmittance thresholds. Figure 2 presents the probability distribution of specific attenuation occurrence against the upper boundaries of grouped specific attenuation. It was observed that the most probable estimated specific attenuation at $650 \mathrm{~nm}$ ranges from $0.00-5.00 \mathrm{~dB} / \mathrm{km}$ with a percentage probability value of about $67.38 \%$. For the $850 \mathrm{~nm}$ wavelength, the same attenuation range is the most probable with a percentage probability of about $75.95 \%$. At the same optical wavelength of $650 \mathrm{~nm}$, the second most probable specific attenuation range from $5.01-10.00 \mathrm{~dB} / \mathrm{km}$, with a percentage probability of about $18.54 \%$. At $1550 \mathrm{~nm}$ wavelength, a similar trend was observed with a percentage probability of about $1.52 \%$ for specific attenuation range from $10.01-15.00 \mathrm{~dB} / \mathrm{km}$. This implies that Akure can be characterized as a low fog-induced specific attenuation location.

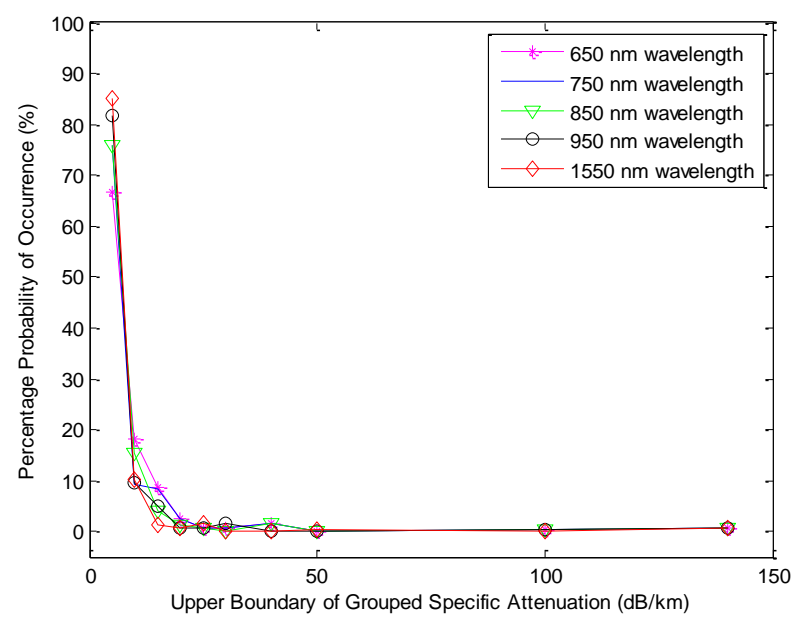

Figure 2. Probability of specific attenuation at different wavelengths and $2 \%$ transmittance thresholds 
Figure 3 presents the estimated rain-induced specific attenuation against rain rates in November and March, which marked the beginning and end of the dry season months respectively. It was observed that the maximum specific attenuation in November is about $18.82 \mathrm{~dB} / \mathrm{km}$ while that of March is about $21.38 \mathrm{~dB} / \mathrm{km}$. Although not shown here, other months in the dry season show similar trend with different values of specific attenuation. Figure 4 also presents the estimated rain-induced specific attenuation against rain rates in April and October, which marked the beginning and end of the raining season months respectively. The maximum estimated specific attenuation in April is about $28.87 \mathrm{~dB} / \mathrm{km}$ while the month of October, the maximum specific attenuation is about $27.98 \mathrm{~dB} / \mathrm{km}$. In terms of peak estimated specific attenuation, the worst month whereby the greatest impairment along the FSO link is May with peak attenuation value of about $34.90 \mathrm{~dB} / \mathrm{km}$. Generally speaking, there are few cases of estimated specific attenuation in order of 20 and $30 \mathrm{~dB} / \mathrm{km}$, yet the overall effect of rain on the proposed link is low due to the low average values in each month of the year.

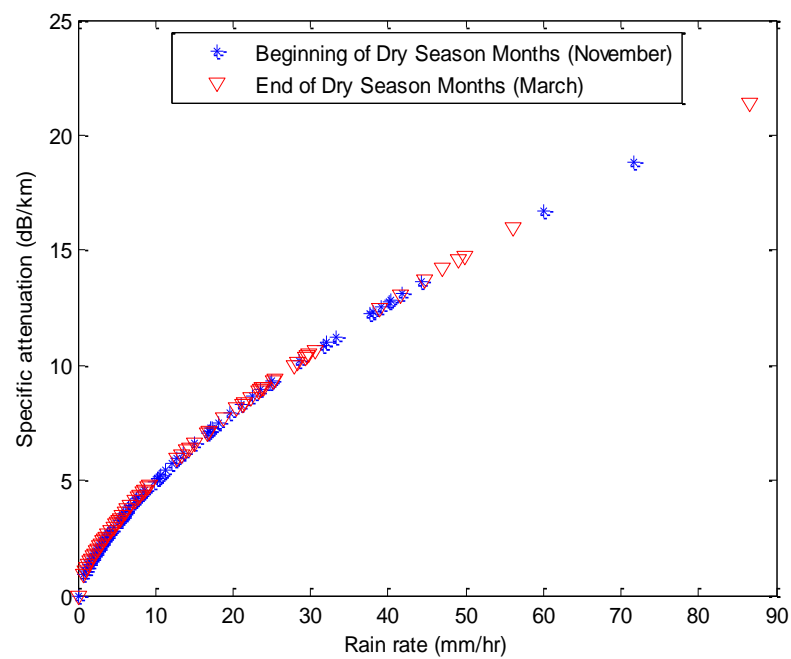

Figure 3. Estimated rain-induced specific attenuation at the beginning and end of dry season months

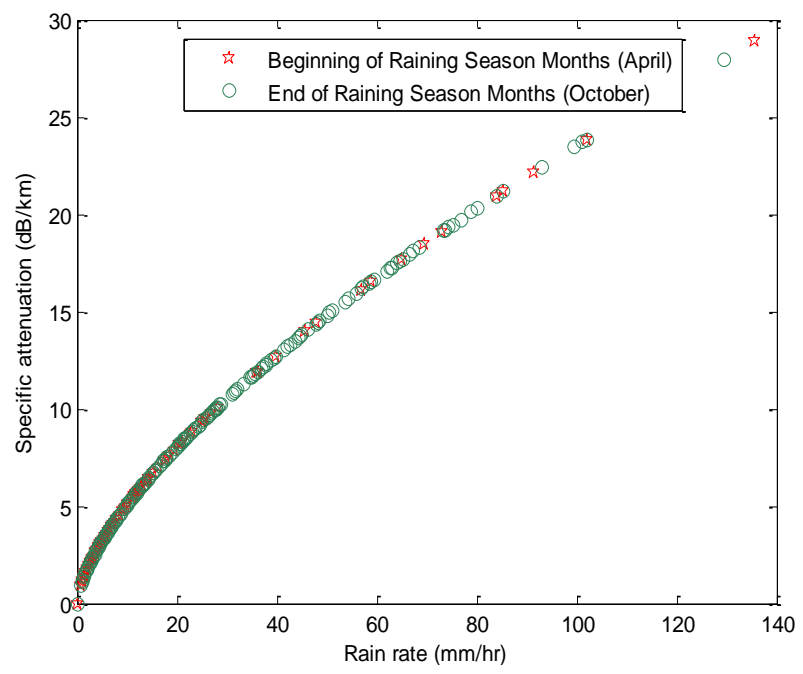

Figure 4. Estimated rain-induced specific attenuation at the beginning and end of raining season months

The expected performance of an FSO system in the study area is evaluated by link margin analysis as simulated using the parameters of Terescope 5000. The first scenario considered in the link margin analysis is the combined effects of geometric loss and atmospheric loss due to reduced visibility.

APTIKOM J. CSIT Vol. 4, No. 1, 2019: $37-44$ 
The results are presented in Figure 5. For example, at $2 \mathrm{~km}$ link distance, the link margins are 4.69, 12.36, $18.23,22.85$ and $38.09 \mathrm{~dB}$ at $650,750,850,950$ and $1550 \mathrm{~nm}$ wavelengths respectively. With the sensitivity of Terescope 5000 being $-46 \mathrm{dBm}$, the FSO link will be available at all five wavelengths at the $2 \mathrm{~km}$ link distance. At $5 \mathrm{~km}$ link distance, the values of link margin are $-76.18,-57.01,-42.35,-30.77$ and $7.30 \mathrm{~dB}$ at $650,750,850,950$ and $1550 \mathrm{~nm}$ wavelengths respectively. This shows that the system will only be available at higher wavelengths of 850,950 and $1550 \mathrm{~nm}$, where the link margin is greater than the receiver sensitivity. The system gets worse at a link range of $10 \mathrm{~km}$ where the link margins are as low as $-214.98 \mathrm{~dB}$ at $650 \mathrm{~nm},-176.63 \mathrm{~dB}$ at $750 \mathrm{~nm},-147.31 \mathrm{~dB}$ at $850 \mathrm{~nm},-124.16 \mathrm{~dB}$ at $950 \mathrm{~nm}$, and -48.01 $\mathrm{dB}$ at $1550 \mathrm{~nm}$ wavelength. At $2 \mathrm{~km}$ link range, the link margin increased by about $711.79 \%$ from $650 \mathrm{~nm}$ to $1550 \mathrm{~nm}$ wavelength while at $5 \mathrm{~km}$ link distance, the increase in link margin between the same set of wavelengths is about $109.59 \%$. Although transmission at higher optical wavelengths reduce attenuation on an FSO link, however these results revealed that the adoption of a higher operating wavelength becomes less important as the link range increases.

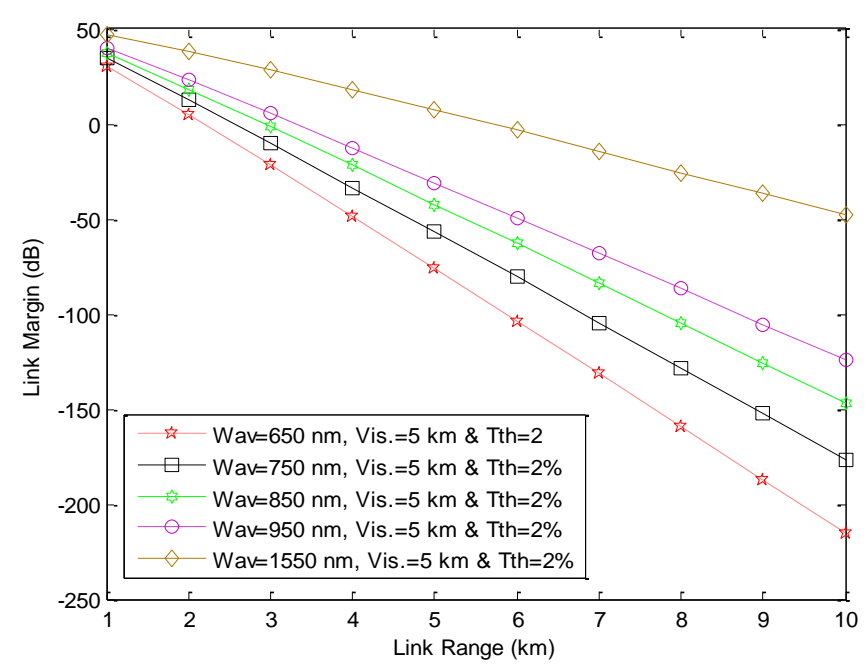

Figure 5. Link margin with geometric and fog-induced loss at $5 \mathrm{~km}$ visibilitytransmittancethreshold

Figure 6 also shows the plots of the link margins due to the combined effects of geometric loss and atmospheric loss due to rain. At $2 \mathrm{~km}$ link distance, the link margins are 55.87, 52.13, 48.98, 46.18, $32.60,15.11$ and $-12.72 \mathrm{~dB}$ at $5,10,15,20,50,100$ and $200 \mathrm{~mm} / \mathrm{hr}$ rain rates respectively. The link remains available at all rain rates (even at $200 \mathrm{~mm} / \mathrm{hr}$ ) since the link margins are greater than the receiver sensitivity of $-46 \mathrm{dBm}$. At $5 \mathrm{~km}$ link range, the link margins are 51.75, 42.40, 34.55, 27.53, -6.41, -50.13 and $-119.70 \mathrm{~dB}$ at the specified rain rates respectively. The link becomes unavailable at higher rain rates of 100 and $200 \mathrm{~mm} / \mathrm{hr}$. At a further link distance of $10 \mathrm{~km}$, the link margins are 40.89, 22.20, 6.49, -7.55, $-75.43,-162.88$, and -302.02 at the specified rain rates. These values again show that the link remains unavailable at rain rates above $50 \mathrm{~mm} / \mathrm{hr}$. At $20 \mathrm{~mm} / \mathrm{hr}$ rain rate, the link margin is reduced by $40.38 \%$ from the $2 \mathrm{~km}$ range to the $5 \mathrm{~km}$ range. From the $5 \mathrm{~km}$ range to the $10 \mathrm{~km}$ range, the link margin further decreases by about $127.43 \%$. At $200 \mathrm{~mm} / \mathrm{hr}$ rain rate, the link margin is reduced by about $841.30 \%$ from the $2 \mathrm{~km}$ range to the $5 \mathrm{~km}$ range, and $152.31 \%$ from the $5 \mathrm{~km}$ range to the $10 \mathrm{~km}$ range. These results show that the percentage decrease in link margin is much higher at higher rain rates and shorter link ranges. 


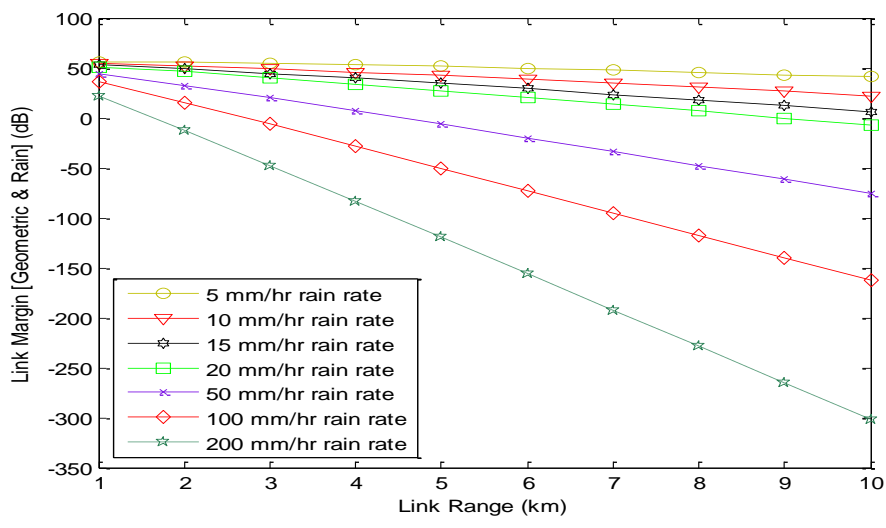

Figure 6. Link margin with geometric and rain losses at different rain rates and link ranges

\section{Conclusion}

In this study, fog- and rain-induced specific attenuations have been investigated for terrestrial FSO communication over Akure, Nigeria. Retrieved one-year archived visibility and rain data have been used to compute both fog- and rain-induced losses at five optical wavelengths of 650, 750, 850, 950 and $1550 \mathrm{~nm}$, at $2 \%$ transmittance thresholds. Results on the fog-induced specific attenuation shows peak values of about $122.14,109.96$ and $86.91 \mathrm{~dB} / \mathrm{km}$ at 650,850 and $1550 \mathrm{~nm}$ wavelengths respectively. The result shows that fog-induced specific attenuation generally decreases with increasing optical wavelength. These maximum values occur thrice throughout the year of study. Although these high values potent danger for an FSO link, however results from the percentage probability of fog-induced specific attenuation occurrence have shown that the most probable specific attenuation ranges from $0.00-5.00$ $\mathrm{dB} / \mathrm{km}$ for all the wavelengths considered. This implies that the study location can be characterized as a low attenuation region, thus very suitable for FSO communication system implementation.

Considering the effect of rain on FSO link shows that the rain-induced specific attenuation are much lower than fog-induced attenuation at the study area. This again reaffirms the characterization of the study area as low attenuation region, thus implying that FSO communication system can be suitably implemented at the study area since it is majorly characterized by rainfall. Further results on the link margin analysis considering geometric and fog-induced losses shows that although transmission at higher optical wavelengths reduce attenuation on an FSO link, however results have revealed that the adoption of a higher operating wavelength for attenuation reduction becomes less effective as the link range increases. The overall result will serve as a good tool for technical choice of FSO link design and the suitable wavelength for initiating point - to - point links in the study area.

\section{References}

[1] Ghassemlooy Z, Popoola W, Rajbhandari S. Optical Wireless Communications-System and Channel Modelling with MATLAB, CRC Press, Taylor \& Francis Group, 6000 Broken Sound Parkway NW, Suite 300 Boca Raton. 2013. FL 33487-2742

[2] M Saleem Awan et al. Attenuation Analysis for Optical Wireless Link Measurements under Moderate Continental Fog Conditions at Milan and Graz. 2008 IEEE 68th Vehicular Technology Conference, Calgary, BC, 2008: 1-5.

[3] Flecker B, Gebhart M, Leitgeb E, Sheikh M, Chlestil C. Results of attenuation measurements for optical wireless channel under dense fog conditions regarding different wavelengths. In Proc. SPIE. 2006; 6303.

[4] Awan MS, Leitgeb E, Horwath LC, Muhammad SS, Nadeem F, Khan MS. Characterization of Fog and Snow Attenuations for Free-Space Optical Propagation, Journal of Communications, 2009; 4 (8): 533-545.

[5] Adimula IA. Characteristics of Rain Induced Attenuation and Phase shift in Ilorin, Nigeria, Zuma Journal of Pure and Applied Science. 2003; 5(2): 193-196.

[6] Hall PM, Braclay LW, Hewitt MT. Propagation of Radiowaves. IEE Press. London. 1996.

[7] Mohamed OT, Mahmud MB, Adam FA. Analysis of Rain Effects on Free Space Optics Based on Data Measured in the Lybyan Climate, International Journal of Information and Electronics Engineering. 2014; 4(6): 469-472.

[8] Rouissat M, Borsali AR, Chikh-bled ME. Free Space Optical Channel Characterization and Modelling with Focus on Algeria Weather Conditions, I. J. Computer Natwork and Information Security. 2012; 3: 17-23.

[9] MRV Communications (2002), Terescope 5000 Datasheet, http://www.mrvfso.com/mrvsupport

APTIKOM J. CSIT Vol. 4, No. 1, 2019: 37 - 44 